\title{
RADIATION DAMPING AND THE TWO MODE BEHAVIOR IN PULSARS
}

\author{
C. -I. BJÖRNSSON \\ Stockholm Observatory
}

\begin{abstract}
It is argued that both observations and theory indicate that radiation damping plays an important rôle in pulsar emission. The two-mode behavior as well as the observed value of the brightness temperature can both be understood as a result of radiation damping. In this context, a possible cause for the enhanced depolarization in the wings of pulsar profiles is discussed.
\end{abstract}

\section{Energy losses in pulsars: local}

In the standard polar cap model (Ruderman and Sutherland 1975) it is assumed that the radiating bunches do not suffer any radiative reaction, i.e. the cooling time is much longer than $\sim \gamma^{2} / \nu$ ("the emission time"), where $\gamma$ is the Lorentz factor of the bunches and $\nu$ the observed frequency. However, this is not necessarily the case.

For a bunch containing $N$ particles and total kinetic energy $E$

$$
\frac{\Delta E}{E}=\frac{2}{3} \frac{r_{0}}{p} \gamma^{3} \Delta \chi N,
$$

where $\Delta E$ is the energy lost by the bunch when traversing an angle $\Delta \chi$ along a magnetic field line with radius of curvature $p$, and $r_{0}$ is the classical electron radius. Assuming that the emission is optically thin, i.e., $\gamma N m c^{2}>k T$, eq.(1) becomes

$$
\frac{\Delta E}{E}>2.2 \times 10^{-33} \frac{T}{\gamma^{2}} \nu_{\mathrm{c}}(\gamma \Delta \chi),
$$

where $T$ is the brightness temperature and $\nu_{\mathrm{c}} \equiv$ $3 c \gamma^{3} / 2 \pi p$. Normally, the observed brightness temperature is calculated using the cone opening angle. This under estimates the real value, since the radiation is emitted locally within a solid angle $\Omega \sim \gamma^{-2}$. Using the definition of $T\left(\equiv L c^{2} / 2 k A \nu^{3} \Omega\right)$ one obtains from eq. (2a)

$$
\frac{\Delta E}{E}>0.5 \frac{L_{28}}{A_{15} \nu_{400}^{2}}(\gamma \Delta \chi),
$$

where $L_{28}$ is the luminosity (in units of $10^{28} \mathrm{erg} / \mathrm{s}$ ) emitted from an area $A_{15}$ (in units of $10^{15} \mathrm{~cm}^{2}$ ) at a frequency $\nu_{400}$ (in units of $400 \mathrm{MHz}$ ). Furthermore, $\Omega=\gamma^{-2}$ has been used. Since $L_{28} \sim 1$ is a typical value for pulsar luminosity at $\nu_{400}=1$ and $A_{15} \sim 1$ from Ruderman and Sutherland, eq.(2b) shows that the cooling time is not longer than the emission time $\left(\Delta E / E>1\right.$ for $\left.\Delta \chi \sim \gamma^{-1}\right)$. This suggests that radiation damping is an important characteristic of pulsar radiation; e.g. it determines the brightness temperature, since the bunches are not expected to grow appreciably after cooling has become important and, as is argued below, the polarization properties differ substantially from those of ordinary curvature radiation.

$\mathrm{Eq} .(2 \mathrm{~b})$ is derived under the assumption that the coherence factor can be obtained by increasing the charge and mass by a factor $N$ in the single particle emission formulæ; e.g. $\nu=\nu_{\mathrm{c}}$ is assumed. However, this may be invalidated either by narrow band emission and/or that the emitted frequency is not $\nu_{\mathrm{c}}$ but rather $\nu<\nu_{\mathrm{c}}$, e.g. in the Ruderman and Sutherland model $\nu \approx \gamma^{1 / 2} \nu_{\mathrm{p}} \leq \nu_{\mathrm{c}}$, where $\nu_{\mathrm{p}}$ is the plasma frequency. In the first case eq. $(2 b)$ has to be multiplied by $\Delta \nu / \nu$, where $\Delta \nu$ is the bandwidth, and in the second case by $\left(\nu / \nu_{\mathrm{c}}\right)^{4 / 3}$, since the single particle flux $\propto \nu^{1 / 3}$. Simultaneous measurements of individual pulses at several frequencies (e.g. Stinebring 1982) show a high degree of correlation both in intensity and polarization, indicating that a substantial fraction of the radiation is broad band. Furthermore, multiplying eq. (2a) by $\left(\nu / \nu_{\mathrm{c}}\right)^{4 / 3}$ gives

$$
\nu(\gamma \Delta \chi)\left(\frac{\nu}{\nu_{\mathrm{c}}}\right)^{4 / 3}=\nu(\gamma \Delta \chi)\left(\frac{\nu}{\nu_{\mathrm{c}}}\right)^{1 / 3} .
$$

Since the "emission time" for $\nu<\nu_{\mathrm{c}}$ corresponds to $\Delta \chi \sim \gamma^{-1}\left(\nu / \nu_{c}\right)^{-1 / 3}$, the conclusion regarding the importance of radiation damping is not changed for $\nu<\nu_{\mathrm{c}}$

\section{Energy losses in pulsars: global}

Ruderman and Sutherland obtained an upper limit of $\sim 10^{30} \mathrm{erg} \mathrm{s}^{-1}$ for the energy, which can be generated by the growth and subsequent break down of a potential gap close to the neutron-star surface by assuming that particles are accelerated continuously in a gap where the potential difference has its largest value possible. Since the observed single pulses do not fill the pulsar window uniformly, the energy generation occurs intermittently. A more realistic (perhaps?) estimate of the energy generation 
rate $(P)$ is obtained from

$$
P \sim \frac{\left\langle E^{2}\right\rangle_{\max }}{8 \pi} \frac{V}{t_{\text {gap }}}
$$

where $\left\langle E^{2}\right\rangle_{\max } / 8 \pi$ is the maximum value of the average electric field energy density in the volume $V$ of the gap. $t_{\text {gap }}$ is the time it takes for the gap to grow and break down. This value of the energy generation rate is smaller by a factor $8 \pi t_{\text {gap }} /(h / c)$ than the maximum one obtained by Ruderman and Sutherland; $h$ is the height of the gap. Ruderman and Sutherland estimate $t_{\text {gap }}$ as a few microseconds; thus this factor is $\sim 10^{2}$ and a "typical" energy generation rate is more like $\sim 10^{28} \mathrm{erg} \mathrm{s}^{-1}$. Since this is equal to a typical pulsar radio luminosity, this suggests that most of the energy generated in the polar gap is radiated away as radio emission. Thus, both local and global considerations indicate that radiation losses are an important aspect of pulsar radiation.

\section{The two-stream instability}

In the two-stream instability the bunches grow until either the cooling time equals the time scale for growth or all the energy contained in the relative motion of the two streams has gone into bunching. The dimensions of the bunches are expected to be equal in all directions in the frame where the instability corresponds to purely growing modes and given by $\sim \gamma \lambda$. The maximum number of particles in a bunch is then (cf. Ruderman and Sutherland 1975)

$$
N_{\max } \sim \frac{E_{\max } \gamma \lambda}{e^{2}},
$$

where $E_{\max }$ is the maximum energy per particle generated by the two-stream instability. With $E_{\max } \sim m c^{2}$, one obtains $N_{\max } \sim \gamma \lambda / r_{0}$. Using $N=N_{\max }$ in eq.(1) gives

$$
\frac{\Delta E}{E(\gamma \Delta \chi)} \sim 1
$$

which shows that the cooling time is approximately equal to the "emission time". Thus, the theory of two-stream instability suggests that radiation damping plays a significant rôle in pulsar radiation. This emphasizes the conclusions reached above from consideration of the observations.

\section{Implications}

The braking gives rise to a parallel (de)acceleration with a characteristic time scale $\sim \gamma^{2} / \nu$; this should be compared with the perpendicular acceleration due to the streaming along curved field lines, which has a characteristic time scale $2 \pi / c \sim \gamma^{3} / \nu$. Since power $\propto(\text { acceleration })^{2}$ and parallel acceleration is a factor $\gamma^{2}$ less efficient than perpendicular acceleration, it is seen that approximately equal power is radiated in the two modes. A similar argument has been applied by Cheng and Ruderman (1979) to the acceleration associated with the formation of the bunches. However, some of the characteristics of braking-radiation differ "favorably" from those of bunching-radiation: (i) It is dipole radiation (not quadrupole as in bunching-radiation) and a phase-coherent mixing between perpendicular and parallel acceleration occurs. This can cause smooth $\sim 90^{\circ}$ swings in the polarization angle without abrupt changes in the intensity (see below). (ii) The observed brightness temperature can be understood as a result of radiation damping.

A correct calculation of the polarization properties has to include the effects of radiation damping self-consistently. Although this has not been done, the qualitative features of the radiation should be similar to those obtained by Björnsson (1984), where a predescribed variation in the velocity was superposed on the constant streaming velocity. The important parameter is

$$
\delta=\alpha \frac{1}{\gamma^{2}}\left(\frac{t_{\perp}}{t_{\|}}\right)^{2},
$$

where $t_{\perp} \sim \gamma^{3} / \nu$ is the time scale for perpendicular acceleration and $t_{\|}$is the corresponding time scale for parallel acceleration. The value of $\alpha$ depends on the details of the streaming and velocity variations, typically $\alpha \sim 1-10$. In the case of radiation damping $t_{\|\|} \sim\left(\gamma^{2} / \nu\right)^{1 / 3}$ and $\delta \sim \alpha\left(\nu / \nu_{\mathrm{c}}\right)^{2 / 3}$ is expected for most of the radiation. As shown by Björnsson, a two-mode behavior is apparent for $\delta$ larger than a few, i.e. a $90^{\circ}$ swing in the position angle occurs in the emission profile. Furthermore, in the expected range of $\delta$ this two-mode behavior occurs without destroying the circular polarization; in fact, circular polarization $\propto \delta^{-1 / 2}$ for $\delta \gg 1$. The two modes both have a high degree of linear polarization; however integrating over the emission profile causes depolarization. This depolarization increases with $\delta$; for $\delta \gg 1$, linear polarization $\propto \hat{\delta}^{-1}$.

\section{A post-cooling scenario}

The evolution of the bunches after cooling can significantly affect the pulsar emission. Although this is a highly non-linear problem and a realistic treatment is beyond the scope of this paper, due to its potential importance, a few possible aspects of this evolution are discussed here.

The streaming particles pass through a bunch in a time $\sim \lambda\left(\gamma^{2} / c\right)$, which is the "emission time". 
Thus, when bunches start to cool, individual particles lose energy while passing through the bunch. A possible post-cooling scenario for the bunches is the following: (i) The Lorentz factor of the bunches decreases. (ii) Since more particles stream into a bunch than leave it (due to cooling), the number of particles in a bunch can become larger than $N_{\max }$. The energy needed for this enhanced bunching is derived from the bulk motion of the stream, not from the relative motion as in the two-stream instability.

In the two-stream instability one expects that the maximum number of particles in a bunch is $\sim\langle n\rangle A_{\perp} \lambda$, where $\langle n\rangle$ is the average number density of streaming particles and $A_{\perp}$ is the surface of the bunch perpendicular to the streaming velocity. Using $A_{\perp} \sim(\gamma \lambda)^{2}$ and the value of $\langle n\rangle$ obtained by Ruderman and Sutherland, one finds $\langle n\rangle A_{\perp} \lambda \sim N_{\max }$, as given in eq.(5). This is consistent with the expectation that when the bunches are cooling, the radiated energy is equal to the rate of kinetic energy flowing into the bunches, i.e.,

$$
\gamma m c^{2} A_{\perp}\langle n\rangle \frac{c}{\gamma^{2}} \sim \frac{r_{0} m c^{3} \gamma^{4} N^{2}}{p^{2}},
$$

leading to $N^{2} \sim\langle n\rangle A_{\perp} \lambda N_{\max }$; hence $N \sim N_{\max }$ when the cooling starts. As $\gamma$ decreases, $N$ becomes larger than $N_{\max }$ and the value of $\delta$ is expected to increase beyond the value given above in the previous section.

This "cooling-radiation" is effective only for $\gamma$ large enough for $\nu \leq \nu_{\mathrm{c}} \propto \gamma^{3}$. Since, initially, $\nu / \nu_{\mathrm{c}}$ decreases with distance away from the cone axis, the "cooling-radiation" is most prominent in the wings of pulsar profiles. Because the effective value of $\delta$ increases with the initial value of $\nu_{\mathrm{c}} / \nu(N$ has more time to grow before $\nu / \nu_{\mathrm{c}}$ occurs), pulsar profiles should show the largest depolarization in the wings. This is consistent with observations. 